\title{
Comparative evaluation of the use of dry binders in a physical mixture or as a coprocessed dry binder in matrix tablets with extended drug release
}

\author{
JITKA MUŽÍKOVÁ ${ }^{1 *}$ \\ ALENA KOMERSOVÁ ${ }^{2}$ \\ VÁCLAV LOCHA ${ }^{2}$ \\ LUCIE VILDOVÁ ${ }^{1}$ \\ BÁRA VOŠOUSTOVÁ ${ }^{2}$ \\ MARTIN BARTOŠ \\ ${ }^{1}$ Department of Pharmaceutical \\ Technology, Charles University \\ Faculty of Pharmacy in Hradec \\ Králové, Czech Republic \\ ${ }^{2}$ Department of Physical Chemistry \\ Faculty of Chemical Technology \\ University of Pardubice \\ Czech Republic \\ ${ }^{3}$ Department of Analytical Chemistry \\ Faculty of Chemical Technology \\ University of Pardubice \\ Czech Republic
}

Accepted April 5, 2018

Published online April 20, 2018

\begin{abstract}
This paper evaluates and compares the properties of directly compressible tabletting materials and matrix tablets containing a combination of $\alpha$-lactose monohydrate and microcrystalline cellulose in the 3:1 ratio in a physical mixture and in a coprocessed dry binder. Tested parameters include flow properties, compressibility, compactibility and the rate of drug release from tablets. Compressibility is evaluated by means of the energy profile of the compression process. Compactibility is evaluated by means of the tensile strength of the tablets. Dissolution testing is done using the rotating basket method. Dissolution profiles are evaluated by non-linear regression analysis.

Total energy of compression and plasticity values were higher in tabletting materials with the coprocessed dry binder. Increasing additions of poly vinyl alcohol decreased the values of total energy of compression, plasticity, tensile strength of tablets and drug release rate. Dissolution behaviour of tablets, which contained the physical mixture or coprocessed dry binder and the same amount of polyvinyl alcohol, was comparable.
\end{abstract}

Keywords: coprocessed dry binder, matrix tablets, polyvinyl alcohol, energy profile of compression, tensile strength of tablets, dissolution testing

Required properties of tablets can be achieved by combinations of excipients. Development of such combinations is a complex of different adjustment processes. The spectrum of functional modifications of excipients can be substantially extended by e.g. coprocessing, which includes interaction of two or more auxiliary substances at the sub-particle level when a homogeneous excipient develops. The whole procedure is aimed at improving functionality. This method also gives rise to coprocessed dry binders, which play a significant role in direct tablet compression (1-3).

\footnotetext{
*Correspondence; e-mail: muzikova@faf.cuni.cz
} 
Coprocessing improves the properties of dry binders such as flowability, compressibility, dilution potential and lubricant sensitivity. Compressibility of several coprocessed excipients such as Cellactose ${ }^{\circledR}(4)$, Prosolv ${ }^{\circledR}$ SMCC $(5,6)$, Ludipress ${ }^{\circledR}(7)$, StarLac ${ }^{\circledR}(8)$ Combilac $^{\circledR}(9)$ has been reported to be superior to the physical mixture of their constituent excipients. The number of advantages of coprocessed dry binders strongly prevails over a few disadvantages. The first problem is that they are substances with a fixed content proportion of components and this ratio is not suitable for the compression of some active ingredients. Also, the cost of these substances is higher due to more demanding preparation $(2,10)$.

Coprocessed dry binders contain, as a rule, a portion of a self-fragmenting substance, which, during compression, forms new surfaces, and substances exhibiting plastic deformation in compression. It is important to select a correct ratio of both substances. The coprocessed dry binder usually contains a larger amount of the fragile substance, which prevents preservation of a disproportionate amount of elastic energy in the tablet and decreases the sensitivity to added lubricants. The principal role of plastically deformable material is the formation of bonds between the particles in the tablet matrix (11). For this reason, a combination of lactose and cellulose is favoured and widely used in coprocessed dry binders. Lactose occurs in coprocessed dry binders most frequently in the form of $\alpha$-lactose monohydrate. The preferred form of cellulose is microcrystalline or powdered cellulose. It advantageously combines positive properties of both components, at the same time masking their undesirable properties. Substances differ in their behaviour under pressure. In compression, cellulose is deformed plastically and primarily provides good binding properties in tablet formation. Lactose is a fragile material, primarily fulfilling the function of filler (12). The most advantageous proportion of these substances found in the tabletting material was lactose/cellulose 3:1 (12). Substances are found in this ratio also in the manufactured coprocessed dry binders MicroceLac ${ }^{\circledR} 100$ (13) and Disintequik ${ }^{\mathrm{TM}}$ MCC 25 (14), which contain microcrystalline cellulose, and Cellactose ${ }^{\circledR} 80$ (15), which contains powdered cellulose.

Use of coprocessed dry binders in matrix tablets with extended drug release is very rarely published. No comparative studies involving the testing of drug release from tablets with coprocessed dry binders have been published to date. This paper is aimed at comparing the properties of directly compressible tabletting materials and matrix tablets containing a combination of $\alpha$-lactose monohydrate and microcrystalline cellulose in the 3:1 ratio in a physical mixture and a coprocessed dry binder. The retarding ingredient employed was polyvinyl alcohol. Properties of the tabletting materials under evaluation included their flow properties, compressibility and compactibility (tensile strength of tablets). Matrix tablets were prepared from the studied tabletting materials and the release rate of model drugs was compared with regard to the composition of tablets.

\section{EXPERIMENTAL}

\section{Materials}

The study used the following substances: coprocessed dry binder MicroceLac ${ }^{\circledR} 100$ (Meggle Pharma, Germany), spray dried lactose Flowlac ${ }^{\circledR} 90$ (Meggle Pharma, Germany), microcrystalline cellulose Comprecel ${ }^{\circledR} 102$ (Mingtai Chemicals Co Ltd, Taiwan) and poly- 
vinyl alcohol Parteck ${ }^{\circledR}$ SRP 80 (Merck KGaA, Germany). Magnesium stearate (Acros organics, USA) was used as lubricant. Salicylic acid (JQC (Huayin) Pharmaceutical Co., Ltd., China) was the model drug.

\section{Preparation of tabletting materials}

The study employed 14 tabletting materials, whose composition is shown in Table I. A mixing cube KB $15 S$ (Erweka $\mathrm{GmbH}$, Germany) was used for the preparation of mixtures. Tabletting materials were prepared by graded mixing. A mixture of dry binders Flowlac 90 and Comprecel 102 in the 3:1 ratio was prepared by mixing the substances for $2.5 \mathrm{~min}$. Mixtures of dry binders with magnesium stearate were prepared by mixing for $2.5 \mathrm{~min}$. Tabletting materials for matrix tablets were prepared by mixing dry binders with Parteck SRP 80 for $2.5 \mathrm{~min}$, and then with salicylic acid for $2.5 \mathrm{~min}$. Finally, magnesium stearate was added for a mixing period of $2.5 \mathrm{~min}$. Tabletting materials intended for the blind sample were mixed in the same manner, omitting the addition of salicylic acid.

\section{Scanning electron microscopy (SEM)}

A compact scanning electron microscope VEGA3 SBU (Tescan, Brno, Czech Republic) was used to study the size, shape and structure of the particles of Flowlac 90, Comprecel

Table I. Composition of tabletting materials

\begin{tabular}{|c|c|c|c|c|c|}
\hline \multirow[b]{2}{*}{ Formulation } & \multicolumn{5}{|c|}{ Tabletting material } \\
\hline & $\begin{array}{l}\text { ML } 100 \\
(\%)\end{array}$ & $\begin{array}{c}\text { F } 90+C 102 \\
(\%)\end{array}$ & $\begin{array}{c}\text { P SRP } 80 \\
(\%)\end{array}$ & $\begin{array}{l}\text { SA } \\
(\%)\end{array}$ & $\begin{array}{c}\text { Mgst } \\
(\%)\end{array}$ \\
\hline $\mathrm{F} 1$ & 99 & & & & 1 \\
\hline F2 & & 99 & & & 1 \\
\hline F3 & 44 & & 30 & 25 & 1 \\
\hline F4 & 34 & & 40 & 25 & 1 \\
\hline F5 & 24 & & 50 & 25 & 1 \\
\hline F6 & & 44 & 30 & 25 & 1 \\
\hline F7 & & 34 & 40 & 25 & 1 \\
\hline F8 & & 24 & 50 & 25 & 1 \\
\hline F9 & 69 & & 30 & & 1 \\
\hline F10 & 59 & & 40 & & 1 \\
\hline F11 & 49 & & 50 & & 1 \\
\hline F12 & & 69 & 30 & & 1 \\
\hline F13 & & 59 & 40 & & 1 \\
\hline F14 & & 49 & 50 & & 1 \\
\hline
\end{tabular}

ML 100 - MicroceLac 100, F 90 - Flowlac 90, C 102 - Comprecel 102, F - formulation, P SRP 80 - Parteck SRP 80, SA - salicylic acid, Mgst - magnesium stearate 
102, MicroceLac 100 and the physical mixture of Flowlac 90 and Comprecel 102 in the 3:1 ratio.

Experimental parameters: acceleration voltage of $15 \mathrm{kV}$, backscattered electron (BSE) detector and low vacuum mode $\left(10 \mathrm{~Pa}, \mathrm{~N}_{2}\right)$. Samples were applied to an aluminium target coated with a carbon adhesive tape (Pelco Tabs ${ }^{\mathrm{TM}}, 12 \mathrm{~mm}$ OD, Ted Pella, Inc., CA, USA). The target was then blown with compressed air and placed into a microscope chamber.

\section{FTIR study of tabletting materials}

IR spectra were measured on a Nicolet 6700 FTIR spectrometer. Spectra were scanned in the wavenumber range from 4000 to $400 \mathrm{~cm}^{-1}$ at spectral resolution of $2 \mathrm{~cm}^{-1}$ and with 64 scans. Samples F1-F14 were prepared using the KBr pellet method.

\section{Evaluation of flowability and Hausner ratio}

Flowability was tested by the flow rate through an orifice, according to the European Pharmacopoeia $9^{\text {th }}$ edition (16), on a Granulate Flow Tester GTB (Erweka, Germany). The time for a powder $(40 \mathrm{~g})$ to flow through a $10 \mathrm{~mm}$ and $15 \mathrm{~mm}$ orifice was determined. All measurements were repeated five times, and the values were averaged. Bulk and tapped densities were measured three times by method I according to the European Pharmacopoeia $9^{\text {th }}$ edition (16), on a Tapped density tester (Erweka, Germany). The Hausner ratio was calculated as the ratio of the average value of tapped and bulk density (16).

\section{Preparation of tablets and energy evaluation of the compression process}

Tablets were compressed on a T1 FRO 50 TH.A1K Zwick/Roell device (Zwick GmbH \& Co. KG, Germany) by means of a special die with a lower and an upper punch. The rate of compression was $40 \mathrm{~mm} / \mathrm{min}$, the preload was $2 \mathrm{~N}$, and the rate of preload was $2 \mathrm{~mm} \mathrm{~s}^{-1}$. The tablets were cylindrical without facets and had a $13-\mathrm{mm}$ diameter and $0.5 \pm 0.001 \mathrm{~g}$ mass. To test lubricant sensitivity of the dry binder, 10 tablets were compressed from dry binders alone and their mixtures with $1 \%$ magnesium stearate at the compression force of $14 \mathrm{kN}$. From the tabletting materials with the retarding component, 10 tablets were compressed at compression forces of 14,16 and $18 \mathrm{kN}$. The press controlling program testXpert V 9.01 (Zwick GmbH \& Co. KG, Germany) calculated the parameters of the "force-displacement" record during tablet preparation. Parameters evaluated were $E_{1}$ - precompression energy $[\mathrm{J}], E_{2}$ - energy of plastic deformation [J], $E_{3}$ - energy of elastic deformation [J], $E_{\max }$ - total energy [J] and Pl - plasticity [\%] $(17,18)$. Furthermore, tablets were also compressed for dissolution tests, always 6 tablets from each tabletting material in such a way that the resulting tensile strength ranged from 0.8-1.0 $\mathrm{MPa}$. For dissolution, 2 tablets from each mixture without the drug were compressed in the same manner for the blind sample.

\section{Evaluation of compactibility}

Compactibility was evaluated by the tensile strength of tablets. Thickness and diameter of 10 tablets were measured with 0.01-mm precision using a Tablet Tester $8 \mathrm{M}$ (Dr. Schleuniger Pharmatron AG, Switzerland). After that, their destruction force in $\mathrm{N}$ was 
measured on the same device. Tablets were tested no sooner than 24 hours after compression. The following equation (Eq. 1) was used to calculate tensile strength according to Fell and Newton (19):

$$
P=2 F /(\pi d h)
$$

where $P[\mathrm{MPa}]$ is the tensile strength of tablets, $F[\mathrm{~N}]$ is the destruction force, $d[\mathrm{~mm}]$ is the diameter and $h[\mathrm{~mm}]$ is the height of tablets.

\section{Evaluation of lubricant sensitivity of dry binders}

Mean values of strengths served to calculate the values of the lubricant sensitivity ratio (LSR), to which the dry binder can be compared as to the standpoint of sensitivity to added lubricants. They can be calculated according to the equation (Eq. 2) (20):

$$
L S R=\left(C_{\mathrm{su}}-C_{\mathrm{sl}}\right) / C_{\mathrm{su}}
$$

where $C_{\mathrm{su}}$ is the strength of tablets without a lubricant and $C_{\mathrm{sl}}$ is the strength of tablets with a lubricant.

In this paper, the values of tensile strength, instead of crushing strength, are used for LSR calculation. Tensile strength is used to increase the precision of evaluation, since tablet dimensions are included in the calculations.

\section{Dissolution studies}

Dissolution testing was performed with a European Pharmacopeia, $9^{\text {th }}$ edition (16), compliant dissolution apparatus (Sotax AT 7 Smart, Allschwil, Switzerland) using the rotating basket method at $100 \mathrm{rpm}$ and $37.0 \pm 0.5^{\circ} \mathrm{C}$ in two different dissolution media (deionized water and simulated gastric fluid). Simulated gastric fluid (SGF) containing $\mathrm{NaCl}, \mathrm{HCl}$, pepsin and deionized water was prepared according to the European Pharmacopeia, $9^{\text {th }}$ edition (16). Six tablets containing salicylic acid and one blank tablet were placed in the baskets and immersed in the dissolution medium $(900 \mathrm{~mL})$. At predetermined times, $3 \mathrm{~mL}$ of the dissolution medium was automatically withdrawn, filtered and then the concentration of salicylic acid was determined by UV VIS spectrometry. Each experiment was performed three times with six tablet samples and one blind tablet. For evaluation of the released amount of salicylic acid, mean values with standard deviations were calculated.

The released amount of salicylic acid was determined by UV VIS spectrometry (spectrophotometer HP Agilent 8453) at $303 \mathrm{~nm}$ using three-point background correction and with a corresponding blind solution. To transform absorbance values into concentrations and percentage, the calibration curve method was used.

\section{Evaluation of dissolution kinetics}

In order to quantitatively evaluate the release of salicylic acid from studied formulations, dissolution profiles were fitted to the first-order kinetic model $(E q .3)(21,22)$ and the Weibull model (Eq. 4) (21): 


$$
\begin{gathered}
A_{\mathrm{t}(1)}=A_{\infty}\left(1-\exp ^{-k_{1} t}\right) \\
A_{\mathrm{t}(1)}=A_{\infty}\left(1-\exp \left[-\lambda\left(t-T_{\mathrm{i}}\right)^{b}\right]\right)
\end{gathered}
$$

where $A_{\mathrm{t}(1)}$ is the amount of drug released in time $t, A_{\infty}$ represents the maximum releasable amount of drug in infinite time (it should be equal to the absolute amount of drug incorporated in solid drug formulation at time $t=0), k_{1}$ is the first-order release rate constant in units of time ${ }^{-1}, \lambda$ represents the reciprocal value of the time scale of the process, the location parameter $T_{\mathrm{i}}$ is the lag time before the onset of dissolution (in most cases zero) and $b$ describes the shape of the dissolution curve progression. When the shape parameter $b$ in $E q .4$ is equal to one, the Weibull empiric model corresponds to the first order kinetic model and parameter $\lambda$ in $E q .4$ corresponds to the first order release rate constant $k_{1}$.

To statistically evaluate the similarity of the dissolution profiles of tablets containing the physical mixture of lactose and microcrystalline cellulose or coprocessed dry binder, the similarity factor $f_{2}$ as a logarithmic transformation of the sum-squared error of the differences between test $T_{\mathrm{j}}$ and reference product $R_{\mathrm{j}}$ was determined according to Eq. 5:

$$
f_{2}=50 \times \log \left\{1+\left(\frac{1}{n} \sum_{j=1}^{n}\left|R_{j}-T_{j}\right|^{2}\right)^{-0.5} \times 100\right\}
$$

where $j$ is the number of samples, $n$ is the number of dissolution sample times (21). Similarity factor $f_{2}$ is 100 when the test and reference profiles are identical and decreases to 0 as the dissimilarity increases. Dissolution profiles of tablets with the physical mixture and the corresponding amount of polyvinyl alcohol were used as the reference $\left(R_{\mathrm{j}}\right)$.

\section{Mathematical and statistical processing of results}

The FTIR spectra of all tabletting materials were processed and normalized by OMNIC software and Origin 9. Energy balance of the compression process was statistically evaluated by the testXpert V 9.01 program (Zwick GmbH \& Co. KG, Germany) directly during the compression process. Results of the tensile strength of tablets were statistically evaluated using Excel. ANOVA $(\alpha=0.05)$ was used for testing statistical significance. All dissolution profiles were evaluated by non-linear regression analysis (Graph Pad Prism 7 and Origin 9 Pro) at a statistical significance level of $p<0.05$.

\section{RESULTS AND DISCUSSION}

The study aims to compare the functionality of a physical mixture of dry binders and a coprocessed dry binder in connection with several parameters of tabletting materials and matrix tablets under study. The coprocessed dry binder was MicroceLac 100, containing $75 \%$ of $\alpha$-lactose monohydrate and $25 \%$ of microcrystalline cellulose. An identical ratio of dry binders was employed to prepare their physical mixture composed of Flowlac 90 
(spray-dried $\alpha$-lactose monohydrate) and Comprecel 102 (microcrystalline cellulose). To characterize the prepared tabletting materials and to exclude the physical or chemical interactions of salicylic acid with cellulose, lactose and polyvinyl alcohol, FTIR spectroscopy was used.

The first part of the study was focused on testing the flow properties of tabletting materials, specifically flowability, bulk and tapped density and determination of the Hausner ratio. These properties were tested both in the physical mixture of dry binders alone and the coprocessed dry binder alone and in directly compressible tabletting materials for matrix tablets containing the retarding component polyvinyl alcohol in three concentrations of 30, 40 and $50 \%$. In dry binders alone, their sensitivity to the lubricant was also determined, specifically at the compression force of $14 \mathrm{kN}$. Directly compressible mixtures with a retarding component were employed to compress tablets with simultaneous evaluation of the energetic profile of the compression process. The tablets then underwent a test for strength. The employed forces of 14,16 and $18 \mathrm{kN}$ were selected in such a way that their tensile strength was, as much as possible, within the range of the optimal strength of 0.56-1.12 MPa (23).

The second part of the study was focused on testing the drug release from matrix tablets. The drug release rate was evaluated based on regression analysis of the dissolution profiles. Tablets intended for the dissolution test were compressed in such a way that their tensile strength ranged from 0.8 to $1.0 \mathrm{MPa}$. Dissolution test was performed by the rotating basket method. Subsequently, kinetics of drug release from all formulations with a retarding component was evaluated.

\section{Evaluation of SEM imaging}

Representative SEM images of Flowlac 90, Comprecel 102, MicroceLac 100 and the physical mixture of Flowlac 90 and Comprecel 102 (in ratio 3:1) are given in Fig. 1. Flowlac 90 is made up of spherical particles with a diameter of 100 to $200 \mu \mathrm{m}$ (Fig. 1a). These particles are a conglomerate of crystals of various sizes with loose pores between them. Particles of MicroceLac 100 have an almost identical size, shape and structure (Fig. 1c). Visually, the porosity of both types of binders seems to be the same. Particles of Comprecel 102 are similar to deformed rods about $150 \mu \mathrm{m}$ in length and about $25 \mu \mathrm{m}$ in width, somewhat resembling "dried tea leaves" (Fig. 1b). A part of these "leaves" is connected to disintegrating aggregates of slightly oval shape and of approximately $200-\mu \mathrm{m}$ length. In the physical mixture of Flowlac 90 and Comprecel 102, undamaged particles of Flowlac 90 are mixed with the "leaves" of Comprecel 102. No aggregates of Comprecel 102 were observed, they were probably broken down during the mixing step (Fig. 1d).

\section{Evaluation of FTIR spectroscopy}

The aim of the FTIR study was to compare the spectra of the physical mixture of lactose and microcrystalline cellulose with those of coprocessed dry binder containing these substances in the same ratio, and to exclude the physical or chemical interactions of salicylic acid with the components of matrix tablets. IR spectra of all formulations were measured.

As it was confirmed that the character of the spectra does not change with increasing concentrations of polyvinyl alcohol in the tabletting materials, only the spectra of the 
J. Mužíková et al.: Comparative evaluation of the use of dry binders in a physical mixture or as a coprocessed dry binder in matrix tablets with extended drug release, Acta Pharm. 68 (2018) 295-311.

tabletting materials containing $30 \%$ of polyvinyl alcohol (F3, F6, F9 a F12) were chosen for more detailed description. These spectra are presented in Fig. 2.

The spectrum of formulation F9 is in good agreement with the spectrum of formulation F12. Both spectra contain a characteristic peak of lactose at $3527 \mathrm{~cm}^{-1}(\mathrm{C}-\mathrm{OH}$ group $)$ and at $1657 \mathrm{~cm}^{-1}\left(\mathrm{C}=\mathrm{O}\right.$ group), and peaks of polyvinyl alcohol between $1740-1710 \mathrm{~cm}^{-1}(\mathrm{C}=\mathrm{O}$ group) $(24,25)$. The bands in F9 and F12 spectra are in good agreement with the bands that were found in the spectra of pure MicroceLac 100 and pure Flowlac 90. Peaks of lactose and polyvinyl alcohol $\left(\mathrm{C}=\mathrm{O}\right.$ group) at $1740-1650 \mathrm{~cm}^{-1}$ are overlapped by strong peaks of salicylic acid ( $\mathrm{C}=\mathrm{O}$ vibration in carboxylic group). It was observed that there were no changes in these main peaks in the spectra of F1-F14.

a)

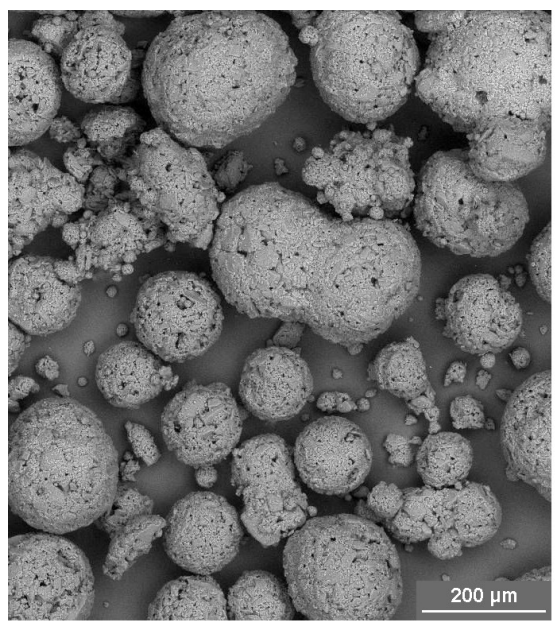

c)

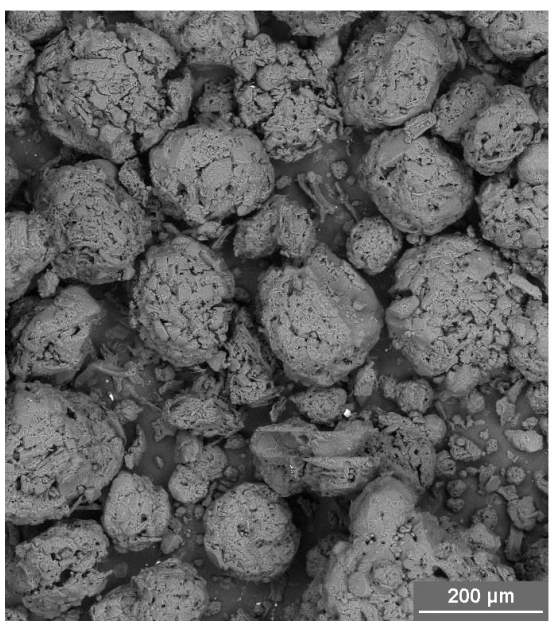

b)

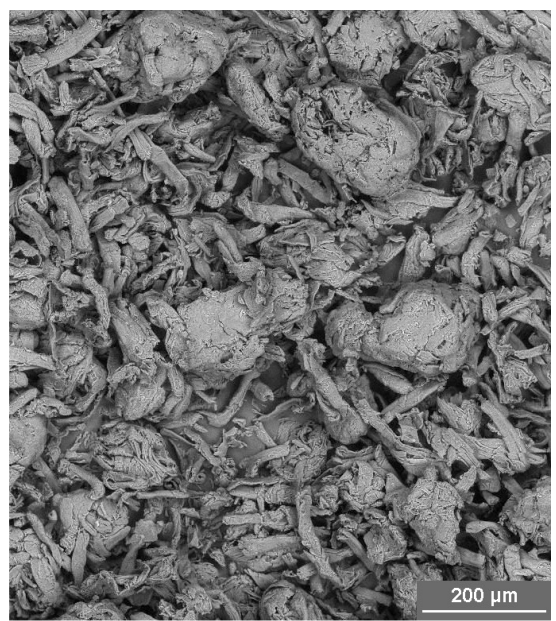

d)

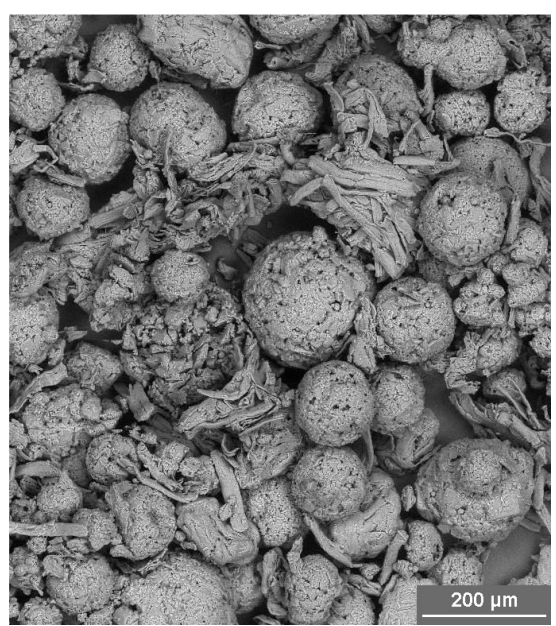

Fig. 1. Representative SEM images of: a) Flowlac 90, b) Comprecel 102, c) MicroceLac 100 and d) physical mixture of Flowlac 90 and Comprecel 102. 


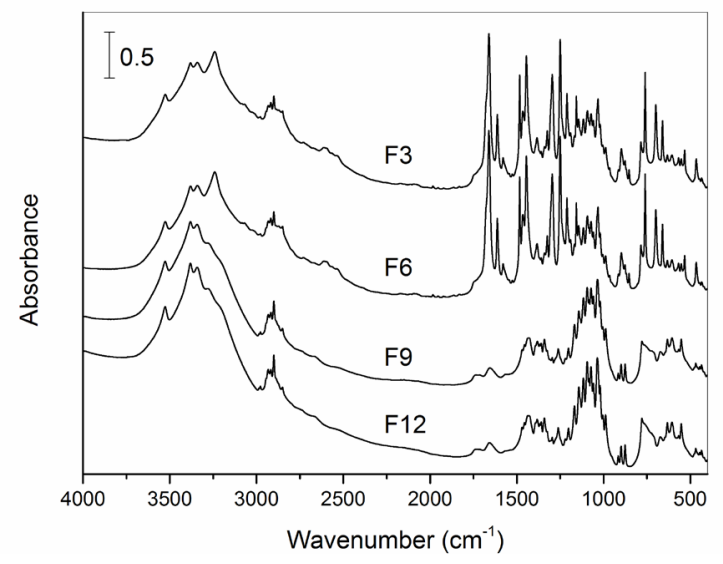

Fig. 2. FTIR spectra of tabletting materials F3, F6, F9, F12.

The FTIR analysis of tabletting materials confirmed that there was no difference in the spectra of materials containing a physical mixture of lactose and microcrystalline cellulose or coprocessed dry binder containing both components mentioned above. Furthermore, the FTIR study revealed no physical or chemical interactions of salicylic acid with cellulose, lactose and polyvinyl alcohol, as evident from Fig. 2.

\section{Evaluation of flow properties}

Flowability of tabletting materials was evaluated in all formulations for matrix tablets and in MicroceLac 100 alone and a physical mixture of Flowlac 90 and Comprecel 102. The results are summarized in Table II. The time during which $40 \mathrm{~g}$ of material flowed off was

Table II. Evaluation of flow properties

\begin{tabular}{cccccc}
\hline $\begin{array}{c}\text { Tabletting } \\
\text { material }\end{array}$ & $\begin{array}{c}\text { Time } \pm \text { SD }(\mathrm{s}) \\
\text { orifice } 1 \mathrm{~cm}\end{array}$ & $\begin{array}{c}\text { Time } \pm \text { SD }(\mathrm{s}) \\
\text { orifice } 1.5 \mathrm{~cm}\end{array}$ & $\begin{array}{c}\mathrm{BD} \pm \mathrm{SD} \\
\left(\mathrm{g} \mathrm{mL}^{-1}\right)\end{array}$ & $\begin{array}{c}\mathrm{TD} \pm \mathrm{SD} \\
\left(\mathrm{g} \mathrm{mL}^{-1}\right)\end{array}$ & HR $\pm \mathrm{SD}$ \\
\hline ML 100 & $6.82 \pm 0.05$ & - & $0.49 \pm 0.01$ & $0.57 \pm 0.00$ & $1.16 \pm 0.01$ \\
F 90 + C 102 & $8.04 \pm 0.26$ & - & $0.49 \pm 0.00$ & $0.63 \pm 0.00$ & $1.29 \pm 0.00$ \\
F3 & Does not flow & $4.08 \pm 0.51$ & $0.61 \pm 0.00$ & $0.74 \pm 0.00$ & $1.21 \pm 0.00$ \\
F4 & Does not flow & $4.68 \pm 0.82$ & $0.60 \pm 0.00$ & $0.75 \pm 0.00$ & $1.25 \pm 0.00$ \\
F5 & Does not flow & Does not flow & $0.58 \pm 0.00$ & $0.75 \pm 0.00$ & $1.29 \pm 0.00$ \\
F6 & Does not flow & $3.38 \pm 0.36$ & $0.59 \pm 0.00$ & $0.75 \pm 0.00$ & $1.27 \pm 0.00$ \\
F7 & Does not flow & $4.52 \pm 0.60$ & $0.59 \pm 0.00$ & $0.75 \pm 0.00$ & $1.27 \pm 0.00$ \\
F8 & Does not flow & Does not flow & $0.507 \pm 0.00$ & $0.77 \pm 0.00$ & $1.35 \pm 0.00$ \\
\hline
\end{tabular}

ML 100 - MicroceLac 100, F 90 - Flowlac 90, C 102 - Comprecel 102, F - formulation, BD - bulk density, TD - tapped density, HR - Hausner ratio, SD - standard deviation 
measured. The first selected orifice had the diameter of $1 \mathrm{~cm}$. Through this orifice, only pure dry binders flowed off gradually. Better flowability versus the physical mixture of dry powders was exhibited by MicroceLac 100 because its flowability time was shorter. Spray drying of two components together further improved the flow rate (26). Tabletting materials with a retarding component did not pass through the $1 \mathrm{~cm}$ orifice, so the 1.5 - $\mathrm{cm}$ orifice was used. With addition of $30 \%$ of polyvinyl alcohol, flowability deteriorated. In this case, worse flowability was found in the mixtures with MicroceLac 100. Differences in flowability results of mixtures with $40 \%$ of polyvinyl alcohol are not statistically significant. Tabletting materials with $50 \%$ of the retarding component did not flow through the $1.5-\mathrm{cm}$ orifice at all. Bulk and tapped densities were also evaluated. Bulk density values were identical in dry binders alone, and tapped density was higher in MicroceLac 100. Addition of the retarding component increased the values of both bulk and tapped density. The best flow was observed in MicroceLac 100 alone, which was also confirmed by the low value of the Hausner ratio (1.16), which classified the character of the flow as good. The physical mixture of dry binders according to the Hausner ratio classified the flow as average (16).

Table III. Values of the energy profils of compression and plasticity $(\mathrm{n}=10)$

\begin{tabular}{ccccccc}
\hline $\begin{array}{c}\text { Tabletting } \\
\text { material }\end{array}$ & $\begin{array}{c}C F \\
(\mathrm{kN})\end{array}$ & $\begin{array}{c}E_{\max } \pm \mathrm{SD} \\
(\mathrm{J})\end{array}$ & $\begin{array}{c}E_{1} \pm \mathrm{SD} \\
(\mathrm{J})\end{array}$ & $\begin{array}{c}E_{2} \pm \mathrm{SD} \\
(\mathrm{J})\end{array}$ & $\begin{array}{c}E_{3} \pm \mathrm{SD} \\
(\mathrm{J})\end{array}$ & $\begin{array}{c}\mathrm{Pl} \pm \mathrm{SD} \\
(\%)\end{array}$ \\
\hline \multirow{2}{*}{$\mathrm{F} 3$} & 14 & $21.67 \pm 0.41$ & $11.395 \pm 0.438$ & $6.808 \pm 0.045$ & $3.465 \pm 0.023$ & $66.27 \pm 0.22$ \\
& 16 & $25.59 \pm 0.42$ & $13.461 \pm 0.396$ & $7.715 \pm 0.079$ & $4.416 \pm 0.023$ & $63.60 \pm 0.28$ \\
& 18 & $29.53 \pm 0.45$ & $15.558 \pm 0.438$ & $8.504 \pm 0.090$ & $5.469 \pm 0.033$ & $60.86 \pm 0.33$ \\
& 14 & $19.80 \pm 0.25$ & $9.472 \pm 0.219$ & $6.832 \pm 0.058$ & $3.496 \pm 0.010$ & $66.15 \pm 0.22$ \\
F4 & 16 & $23.43 \pm 0.23$ & $11.311 \pm 0.213$ & $7.658 \pm 0.086$ & $4.570 \pm 0.061$ & $63.21 \pm 0.35$ \\
& 18 & $27.34 \pm 0.29$ & $13.324 \pm 0.267$ & $8.468 \pm 0.068$ & $5.549 \pm 0.036$ & $60.41 \pm 0.30$ \\
& 14 & $18.76 \pm 0.22$ & $8.463 \pm 0.137$ & $6.749 \pm 0.103$ & $3.544 \pm 0.042$ & $65.57 \pm 0.33$ \\
F5 & 16 & $22.21 \pm 0.27$ & $10.161 \pm 0.188$ & $7.554 \pm 0.178$ & $4.494 \pm 0.051$ & $62.69 \pm 0.65$ \\
& 18 & $25.72 \pm 0.21$ & $11.945 \pm 0.214$ & $8.214 \pm 0.052$ & $5.562 \pm 0.038$ & $59.63 \pm 0.24$ \\
& 14 & $19.94 \pm 0.24$ & $9.786 \pm 0.242$ & $6.647 \pm 0.034$ & $3.506 \pm 0.028$ & $65.47 \pm 0.17$ \\
F6 & 16 & $23.92 \pm 0.39$ & $11.935 \pm 0.388$ & $7.493 \pm 0.051$ & $4.493 \pm 0.049$ & $62.51 \pm 0.24$ \\
& 18 & $27.52 \pm 0.35$ & $13.756 \pm 0.288$ & $8.231 \pm 0.091$ & $5.531 \pm 0.062$ & $59.81 \pm 0.44$ \\
& 14 & $19.43 \pm 0.32$ & $9.155 \pm 0.243$ & $6.743 \pm 0.126$ & $3.534 \pm 0.024$ & $65.61 \pm 0.40$ \\
F7 & 16 & $23.10 \pm 0.25$ & $11.049 \pm 0.202$ & $7.546 \pm 0.073$ & $4.502 \pm 0.054$ & $62.63 \pm 0.38$ \\
& 18 & $26.81 \pm 0.31$ & $12.983 \pm 0.278$ & $8.253 \pm 0.084$ & $5.571 \pm 0.029$ & $59.70 \pm 0.28$ \\
& 14 & $18.02 \pm 0.15$ & $7.930 \pm 0.160$ & $6.549 \pm 0.064$ & $3.539 \pm 0.008$ & $64.92 \pm 0.25$ \\
& 16 & $21.60 \pm 0.17$ & $9.717 \pm 0.171$ & $7.390 \pm 0.092$ & $4.491 \pm 0.034$ & $62.20 \pm 0.27$ \\
& 18 & $25.48 \pm 0.22$ & $11.746 \pm 0.204$ & $8.134 \pm 0.100$ & $5.601 \pm 0.053$ & $59.22 \pm 0.36$ \\
\hline
\end{tabular}

$C F$ - compression force, $E_{\max }$ - total energy, $E_{1}$ - energy of precompression, $E_{2}$ - energy of plastic deformation, $E_{3}$ - energy of elastic deformation, $\mathrm{Pl}$ - plasticity, SD - standard deviation 
Formulations with MicroceLac 100 and 30 and $40 \%$ of polyvinyl alcohol (F3-F4) showed adequate flows, formulations F5-F7 average. The highest value of the Hausner ratio (1.35), with the flow character classified as bad, was recorded in formulation F8 (physical mixture of dry binders with $50 \%$ of polyvinyl alcohol). Flowability is the ability of powders to flow vertically under defined conditions. The Hausner ratio evaluates flow properties, which lead to the classification of compressibility (the ratio of tapped to bulk density). It is the reason why these results do not correlate with each other in some cases.

\section{Evaluation of the compression process energy profile}

Values of the energy profile of the compression process are tabulated in Table III. The total energy of the compression process $E_{\max }$ increases with compression force and their values are higher for tabletting materials with the coprocessed dry binder. The values of this energy decrease with increasing additions of polyvinyl alcohol in both types of tabletting materials. An identical course of dependence is shown by the values of the energy of precompression $E_{1}$. Values of the energy of plastic deformation $E_{2}$, which is important for the formation of bonds, increase also with the compression force and are slightly higher for tabletting materials with the coprocessed dry binder. Addition of polyvinyl alcohol influences these values only in the case of tabletting materials with the physical mixture of dry binders at the concentration of $50 \%$, when the $E_{2}$ value is lower. This fact holds true also for the tabletting material with MicroceLac 100, but only in the case of the compression force of $18 \mathrm{kN}$. Values of the energy of elastic deformation $E_{3}$ increase also with compression force and in the tabletting material with MicroceLac 100 they are slightly increased with increasing additions of polyvinyl alcohol. Plasticity values decrease with compression force and its higher values were recorded in the case of tabletting materials with the coprocessed dry binder. Plasticity values decrease with addition of polyvinyl alcohol, except for the tabletting materials containing the physical mixture of dry binders and $40 \%$ of polyvinyl alcohol. The value in this tabletting material is not statistically significantly different from the value for the tabletting material with the physical mixture of dry binders and $30 \%$ of polyvinyl alcohol.

\section{Evaluation of lubricant sensitivity of dry binders and tensile strength of tablets}

In the coprocessed dry binder alone and the physical mixture of dry binders, sensitivity to the addition of the lubricant $1 \%$ magnesium stearate at the compression force of 14 $\mathrm{kN}$ was evaluated. The found LSR value for MicroceLac 100 was 0.37 , and for the physical mixture of dry binders, it was 0.21 . It follows from the above-mentioned values that MicroceLac 100 is more sensitive to lubricant addition than the mixture of the respective dry binders, as the more the LSR value approaches the value of 1 , the higher is the sensitivity (20). This is connected with better flowability of MicroceLac 100. The film of lubricant on the particles is formed during mixing more perfectly than in the case of the physical mixture of dry binders.

In the tabletting materials, tensile strength was evaluated for matrix tablets in dependence on the compression force (Fig. 3). Tensile strength of tablets increased with compression force and its values were higher in the case of tabletting materials with MicroceLac 100. As a spray dried product, it has good deformation properties and has stronger bonds 


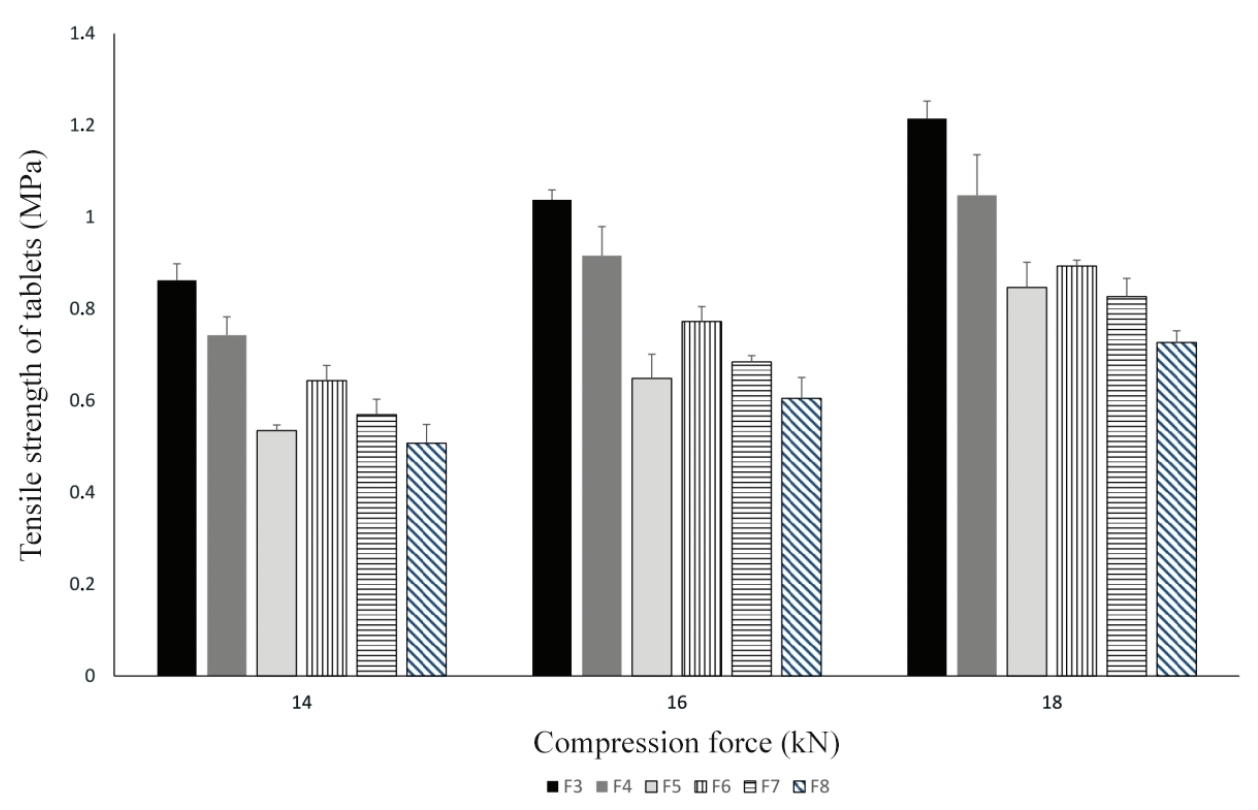

Fig. 3. Tensile strength of tablets in the function of compression force (mean $\pm \mathrm{SD} ; n=10$ ).

with drug particles. The porous surface is more advantageous for multiple interparticle contacts (26). Higher tensile strength is connected with higher values of the energy of plastic deformation E2, which is accumulated in tablets after the compression. Higher values of this energy lead to stronger bonds between the particles. Increasing additions of polyvinyl alcohol decreased the tablet strength, more markedly in the case of tabletting materials with MicroceLac 100.

\section{Evaluation of dissolution studies}

Release rate of the model drug from prepared matrix tablets was evaluated based on regression analysis of the dissolution profiles. Dissolution tests were performed in two different dissolution media - deionized water and simulated gastric fluid (SGF). The first series of experiments was done in deionized water to assess the tablet behaviour in an aqueous medium and further experiments were performed in SGF. The use of SGF for evaluation of the drug release rate in vitro allows, to some extent, to predict the drug behaviour in vivo. SGF is a synthetic form of the gastric fluid which simulates the gastric conditions in the fasted state. SGF is also medium with higher ionic strength in comparison with deionized water, which can affect drug solubility and consequently the drug release rate.

It was found for the dissolution tests performed in deionized water that for both types of tablets (with coprocessed dry binder or with physical mixture) the drug release rate decreases with increasing the amount of polyvinyl alcohol in tablets and there is no difference between these types of tablets with the same content of polyvinyl alcohol (Fig. 4). 
J. Mužíková et al.: Comparative evaluation of the use of dry binders in a physical mixture or as a coprocessed dry binder in matrix tablets with extended drug release, Acta Pharm. 68 (2018) 295-311.

Table IV. Similarity factor $f_{2}$ calculated for the dissolution profiles of tablets containing the physical mixture or coprocessed dry binder and the same amount of polyvinyl alcohol

\begin{tabular}{cccc}
\hline Tablets & $\begin{array}{c}\text { P SRP } 80 \\
(\%)\end{array}$ & $\begin{array}{c}f_{2} \\
\text { deionized water }\end{array}$ & $\begin{array}{c}f_{2} \\
\text { SGF }\end{array}$ \\
\hline F3 vs. F6 & 30 & 85 & 65 \\
F4 vs. F7 & 40 & 85 & 57 \\
F5 vs. F8 & 50 & 91 & 84 \\
\hline
\end{tabular}

$f_{2}-$ similarity factor calculated according to Eq. (5).

Minor differences in the dissolution behaviour between tablets containing the physical mixture or coprocessed dry binder and the same amount of polyvinyl alcohol are statistically insignificant. As can be seen from Table IV, the $f_{2}$ metrics values of 85,85 and $91 \%$ (30, 40 and $50 \%$ of polyvinyl alcohol) also show similarity of the dissolution profiles of tablets containing the physical mixture or coprocessed dry binder and the same amount of polyvinyl alcohol. Similarity of these dissolution profiles is also confirmed by the kinetic parameters obtained from the non-linear regression analysis of the dissolution profiles (Table V). From the values of the coefficient of determination $\left(R^{2}\right)$ and the sum of squares of residues (RSS), it is obvious that all studied formulations meet the Weibull model better than the first-order kinetic model. As can be seen from the values of coefficient $b$ in the Weibull model (Table $\mathrm{V}$ ), for tablets containing the physical mixture and 30 $\%$ of polyvinyl alcohol, the coefficient $b=1.031 \pm 0.046$; it means the Weibull empiric model corresponds to the first order kinetic model, the active ingredient is released on the basis of the first-order kinetics and parameter $\lambda$ corresponds to the first-order release rate constant. From Fig. 3, it is also obvious that the total amount of drug is released from tablets

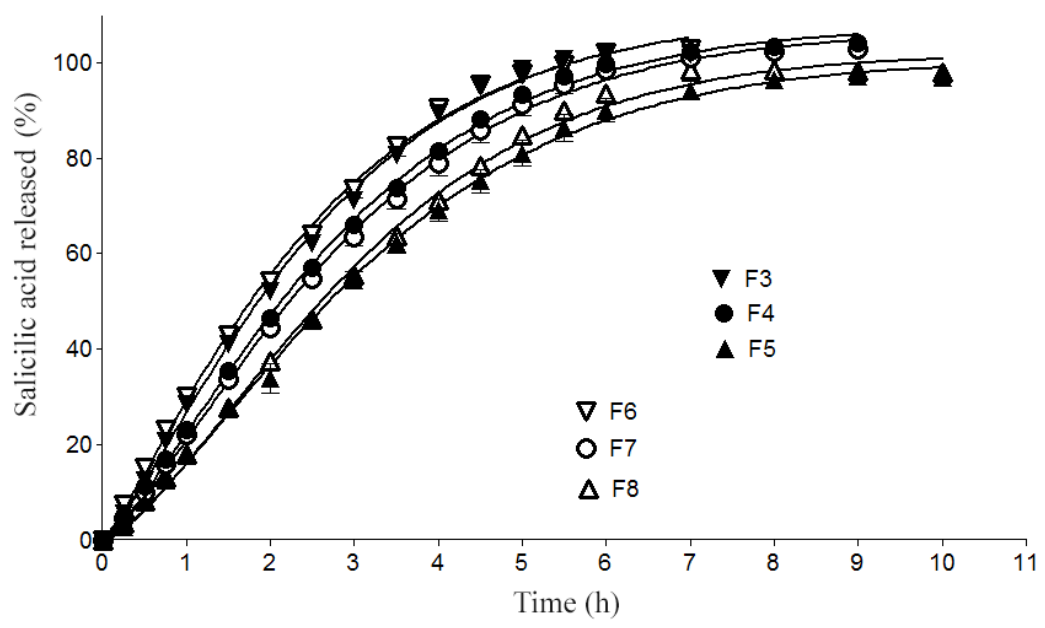

Fig. 4. Dissolution profiles of F3 - F8 formulations in deionized water fitted to the Weibull model (mean $\pm \mathrm{SD} ; n=6)$. 
J. Mužíková et al.: Comparative evaluation of the use of dry binders in a physical mixture or as a coprocessed dry binder in matrix tablets with extended drug release, Acta Pharm. 68 (2018) 295-311.

Table V. Non-linear regression analysis of the dissolution profiles in deionized water - fitted to the first order kinetic model and Weibull model $(\mathrm{n}=6)$

\begin{tabular}{cccccccc}
\hline & \multicolumn{3}{c}{ First-order } & & \multicolumn{5}{c}{ Weibull } \\
\cline { 2 - 8 } Tablets & $k_{1} \pm \mathrm{SD}$ & $\mathrm{RSS}$ & $R^{2}$ & $\lambda \pm \mathrm{SD}$ & $b \pm \mathrm{SD}$ & RSS & $R^{2}$ \\
\hline F3 & $0.2124 \pm 0.0103$ & 668 & 0.9935 & $0.2521 \pm 0.0090$ & $1.160 \pm 0.033$ & 515 & 0.9950 \\
F4 & $0.2076 \pm 0.0101$ & 1234 & 0.9898 & $0.2270 \pm 0.0060$ & $1.249 \pm 0.033$ & 752 & 0.9938 \\
F5 & $0.2087 \pm 0.0110$ & 3520 & 0.9746 & $0.1737 \pm 0.0082$ & $1.389 \pm 0.045$ & 1978 & 0.9857 \\
F6 & $0.2114 \pm 0.0142$ & 563 & 0.9924 & $0.2273 \pm 0.0263$ & $1.031 \pm 0.046$ & 591 & 0.9926 \\
F7 & $0.1887 \pm 0.0121$ & 1810 & 0.9848 & $0.2118 \pm 0.0078$ & $1.259 \pm 0.045$ & 1323 & 0.9889 \\
F8 & $0.2170 \pm 0.0100$ & 2832 & 0.9804 & $0.1721 \pm 0.0052$ & $1.432 \pm 0.029$ & 851 & 0.9941 \\
\hline
\end{tabular}

$k_{1}$ - first order release rate constant, $\lambda$ - reciprocal value of time scale of the process, $T_{\mathrm{i}}$ - location parameter, $b$-shape parameter, RSS - residual sum of squares, SD - standard deviation, $R^{2}-$ coefficient of determination

Table VI. Non-linear regression analysis of the dissolution profiles in SGF-fitted to the first order kinetic model and Weibull model $(\mathrm{n}=6)$

\begin{tabular}{cccccccc}
\hline & \multicolumn{3}{c}{ First-order } & & & Weibull \\
Tablets & $k_{1} \pm \mathrm{SD}$ & $\mathrm{RSS}$ & $R^{2}$ & $\lambda \pm \mathrm{SD}$ & $b \pm \mathrm{SD}$ & RSS & $R^{2}$ \\
\cline { 2 - 8 }$\left(\mathrm{h}^{-1}\right)$ & & & 0.9810 & $0.1938 \pm 0.0034$ & $1.412 \pm 0.017$ & 641 & 0.9973 \\
& F3 & $0.2830 \pm 0.0067$ & 4439 & 0.912 & & \\
F4 & $0.1838 \pm 0.0086$ & 2678 & 0.9709 & $0.1511 \pm 0.0114$ & $1.188 \pm 0.055$ & 2315 & 0.9752 \\
F5 & $0.1678 \pm 0.0061$ & 7021 & 0.9688 & $0.0983 \pm 0.0050$ & $1.437 \pm 0.035$ & 2923 & 0.9870 \\
F6 & $0.3389 \pm 0.0075$ & 3848 & 0.9805 & $0.2747 \pm 0.0079$ & $1.257 \pm 0.029$ & 2342 & 0.9881 \\
F7 & $0.2359 \pm 0.0070$ & 5319 & 0.9731 & $0.1497 \pm 0.0054$ & $1.427 \pm 0.030$ & 1752 & 0.9911 \\
F8 & $0.1801 \pm 0.0051$ & 4095 & 0.9793 & $0.1135 \pm 0.0033$ & $1.390 \pm 0.021$ & 1018 & 0.9949 \\
\hline
\end{tabular}

$k_{1}$ - first order release rate constant, $\lambda$ - reciprocal value of time scale of the process, $T_{\mathrm{i}}$ - location parameter, $b-$ shape parameter, RSS - residual sum of squares, SD - standard deviation, $R^{2}-$ coefficient of determination

containing $30 \%$ of polyvinyl alcohol in about 5.5 hours, from tablets containing $40 \%$ of polyvinyl alcohol in about 7 hours and from tablets with $50 \%$ of polyvinyl alcohol only about $96 \%$ of drug is released in 8 hours. A more detailed evaluation of the mechanism of drug release can be done, for example, based on the Korsmeyer-Peppas model (21).

Like in deionized water, it was confirmed in SGF that for both types of tablets (with coprocessed dry binder or with physical mixture) the drug release rate decreases with increasing amount of polyvinyl alcohol (Fig. 5). As can be seen in Fig. 4, for formulations containing $50 \%$ of polyvinyl alcohol, there are minor differences $\left(f_{2}=84 \%\right)$ between the dissolution profiles of tablets with the physical mixture and with coprocessed dry binder. 


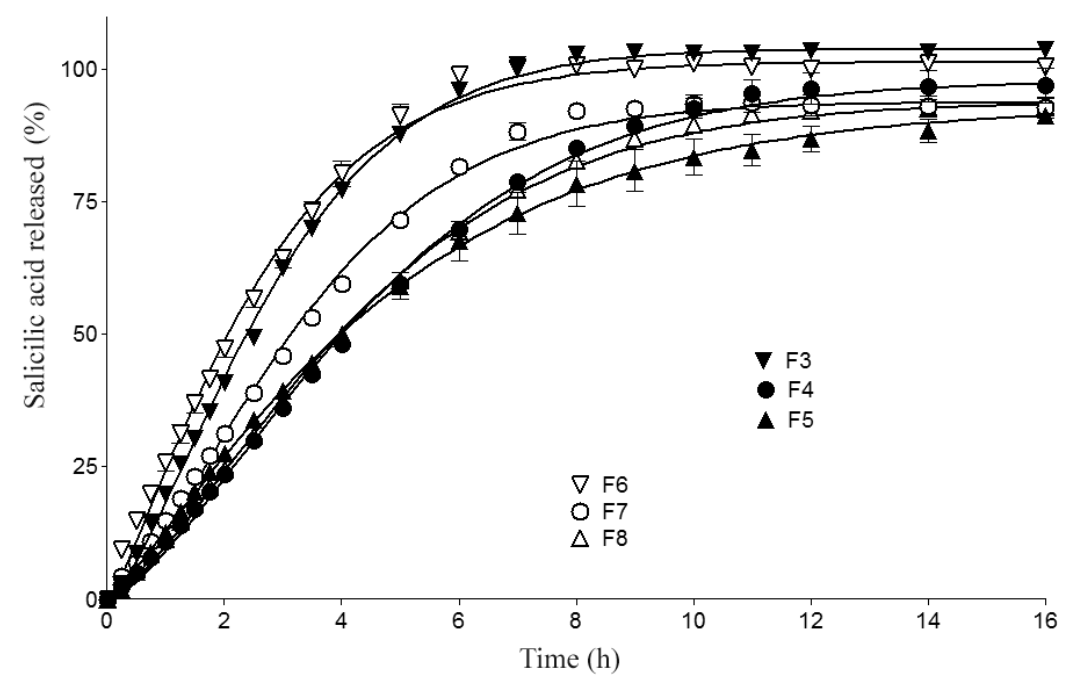

Fig. 5. Dissolution profiles of F3-F8 formulations in SGF fitted to the Weibull model (mean \pm SD; $n=6$ ).

For formulations with lower content of polyvinyl alcohol (30 and $40 \%$ ), the differences in the dissolution behaviour are more pronounced (Fig. 5) but $f_{2}$ values of 65 and $57 \%$ (Table IV) confirmed that these profiles are similar. From formulations containing the physical mixture of lactose and microcrystalline cellulose, salicylic acid is released faster in comparison with formulations with the coprocessed dry binder. The higher release rate of salicylic acid from formulations containing the physical mixture of lactose and microcrystalline cellulose can be affected not only by tablet composition but also by increased ionic strength of the dissolution medium. Complete release achieved for formulations containing $30 \%$ of polyvinyl alcohol compared to lower release for formulations with 40 and 50 $\%$ of polyvinyl alcohol is obvious from Fig. 5. The increasing content of PVA in the tablet prevents the penetration of water into the inner layers of the tablet, resulting in a decreasing amount of water towards the tablet core. A direct consequence of this phenomenon is the decrease of the solubility of salicylic acid and consequently a smaller amount of salicylic acid released. The effect is more pronounced in the acidic dissolution medium in which the solubility of salicylic acid is lower than in water (27). It follows from the values of $R^{2}$ and RSS (Table VI) that the dissolution profiles of all studied formulations meet the Weibull model better than the first-order kinetic model. The first-order kinetic model does not reveal a lower drug release rate at the beginning of the dissolution process.

\section{CONCLUSIONS}

The results show the evident difference in flow properties, compressibility and compactibility of tabletting materials containing the coprocessed dry binder or the physical mixture of dry binders. From the standpoint of flow properties, the best flow was exhibited by MicroceLac 100 alone, which possessed the shortest period of flow through the orifice of $1 \mathrm{~cm}$ and the lowest value of the Hausner ratio of 1.16. The total energy of the 
compression process increased with compression force, its values were higher in the tabletting materials with the coprocessed dry binder. This trend is primarily due to the values of the energy of precompression $\left(E_{1}\right)$. Plasticity decreased with compression force, its values were higher in the tabletting materials with MicroceLac 100. MicroceLac 100 exhibited higher lubricant sensitivity, but at the same compression force it yielded stronger tablets than the mixture of dry binders. Increasing additions of polyvinyl alcohol deteriorated the flowability and decreased the values of the total energy of compression, plasticity and tensile strength of tablets.

Based on regression analysis of the dissolution profiles, it was found that for both types of tablets (with coprocessed dry binder or with physical mixture) the drug release rate decreases with increasing amount of polyvinyl alcohol in tablets. The dissolution behaviour of both types of tablets is comparable. More significant differences in the dissolution profiles between tablets containing the physical mixture or coprocessed dry binder and the same amount of polyvinyl alcohol were found only in simulated gastric fluid for tablets with 30 and $40 \%$ of polyvinyl alcohol.

Acknowledgements. - We thank Meggle Pharma and Merck KGaA for providing the excipient samples used in this study.

\section{REFERENCES}

1. P. Gupta, S. K. Nachaegari and A. K. Bansal, Improved Excipient Functionality by Coprocessing, in Excipient Development for Pharmaceutical Biotechnology and Drug Delivery Systems (Eds. K. A. Katdare, M. V. Chaubal) Informa Healthcare USA 2006, pp. 109-126.

2. K. Satish, S. K. Nachaegari and A. K. Bansal, Coprocessed excipients for solid dosage forms, Pharm. Tech. 28 (2004) 52-64.

3. M. C. Gohel and P. D. Jogani, A review of coprocessed directly compressible excipients, J. Pharm. Pharm. Sci. 8 (2005) 76-93; https://doi.org/10.12691/ajmsm-2-4-2

4. P. M. Belda and J. B. Mielck, The tabletting behavior of Cellactose compared with mixtures of celluloses with lactoses, Eur. J. Pharm. Biopharm. 42 (1996) 325-330.

5. J. D. Allen, Improving DC with SMCC, Manuf. Chemist. 67 (1996) 19-20.

6. B. E. Sherwood and J. W.Becker, A new class of high-functionality excipients: Silicified microcrystalline cellulose, Pharm. Technol. 22 (1998) 78-88.

7. P. C. Schmidt and C. J. Rubensdorfer, Evaluation of Ludipress as a multipurpose excipient for direct compression. Part 1. Powder characteristics and tabletting properties, Drug Dev. Ind. Pharm. 20 (1994) 2899-2925; https://doi.org/10.3109/03639049409042687

8. K. G. Wagner and J. A. Dressler, A corn starch/alpha -lactose monohydrate compound as a new directly compressible excipient, Pharm. Ind. 64 (2002) 992-999.

9. J. Mužíková, P. Svačinová and A. Srbová, A study of a novel coprocessed dry binder composed of $\alpha$-lactose monohydrate, microcrystalline cellulose and corn starch. Pharm. Dev. Technol. 22 (2017) 964-971; https://doi.org/10.3109/10837450.2015.1131717

10. H. Kathpalia and K. Jogi, Coprocessed excipients - a review, WJPR 3 (2014), 3863-3885.

11. G. K. Bolhuis and N. A. Armstrong, Excipients for Direct Compaction - an update, Pharm. Dev. Technol. 11 (2006) 111-124; https://doi.org/10.1080/10837450500464255

12. J. S. M. Gar and M. H. Rubinstein, Compaction properties of cellulose-lactose direct-compression excipient, Pharm. Tech. Int. 15 (1991) 24-27. 
13. Meggle Excipients \& Technology, Technical Brochure MicroceLac ${ }^{\circledR} 100$. Firm. Lit. http://www.megglepharma.com/en/lactose/13-microcelac-100.html, access date March 25, 2015.

14. Kerry, Product document. Disintequik ${ }^{T M}$ MCC 25. Firm. Lit. http://www.sheffieldbioscience.com/disintequik_MCC25/, access date March 28, 2015.

15. Meggle Excipients \& Technology. Technical Brochure Cellactose ${ }^{\circledR}$ 80. Firm. Lit. https://www.megglepharma.com/en/lactose/12-cellactose-80.html, access date October 13, 2017.

16. European Pharmacopoeia, $9^{\text {th }}$ ed., Council of Europe, Strasbourg, 2017.

17. G. Ragnarsson, Force-displacement and Network Measurements, in Pharmaceutical Powder Compaction Technology (Eds. G. Alderborn and Ch. Nyström), Marcel Dekker Inc., New York 1996, pp. 77-96.

18. A. Stamm and C. Mathis, Verpressbarkeit von Festen Hilfsstoffen für Direkttablettierung, Acta Pharm. Technol. 22 (1976) 7-16.

19. J. T. Fell and J. M. Newton, Determination of tablet strength by diametral-compression test, J. Pharm. Sci. 59 (1970) 688-691; https://doi.org/10.1002/jps.2600590523

20. C. E. Bos, G. K. Bolhuis, H. van Doorne and C. F. Lerk, Native starch in tablet formulations: properties on compaction, Pharm. Weekbl. Sci. 9 (1987) 274-282; https://doi.org/10.1007/BF01953630

21. P. Costa and J. M. Sousa Lobo, Modeling and comparison of dissolution profiles, Eur. J. Pharm. Sci. 13 (2001) 123-133; https://doi.org/10.1016/S0928-0987(01)00095-1

22. S. Dash, P. N. Murthy, L. Nath and P. Chowdhury, Kinetic modeling on drug release from controlled drug delivery systems, Acta Pol. Pharm. 67 (2010) 217-223; https://doi.org/10.1080/10717540490265379

23. V. A. Belousov, Choice of optimal pressure values in tabletting medicinal powders, Khim. Farm. Zh. 10 (1976) 105-111.

24. Y. Lei, Q. Zhou, Y. Zhang, J. Chen, S. Sun and I. Noda, Analysis of crystallized lactose in milk powder by Fourier-transform infrared spectroscopy combined with two-dimensional correlation infrared spectroscopy, J. Mol. Struct. 974 (2010) 88-93; https://doi.org/10.1016/j.molstruc.2009.12.030

25. H. S. Mansur, C. M. Sadahira, A. N. Souza and A. A. P. Mansur, FTIR spectroscopy characterization of poly (vinyl alcohol) hydrogel with different hydrolysis degree and chemically crosslinked with glutaraldehyde, Mater. Sci. Eng. C 28 (2008) 539-548; https://doi.org/10.1016/j.msec.2007.10.088

26. A. Michoel, P. Rombaut and A. Verhoye, Comparative evaluation of co-processed lactose and microcrystalline cellulose with their physical mixtures in the formulation of folic acid tablets, Pharm. Dev. Technol. 7 (2002) 79-87; https://doi.org/10.1081/PDT-120002233

27. A. T. M. Serajuddin and C. I. Jarowski, Effect of diffusion layer $\mathrm{pH}$ and solubility on the dissolution rate of pharmaceutical bases and their hydrochloride salts. II: salicylic acid, theophylline and benzoic acid, J. Pharm. Sci. 74 (1985) 148-154; https://doi.org/10.1002/jps.2600740209 